\section{Chronic eosinophilic leukemia with FIP1L1-PDGFRA transcripts after occupational and therapeutic exposure to radiation}

\author{
Gueorgui Balatzenko, 1,4 \\ Nikolay Stoyanov, 2,4 Elena Bekrieva, ${ }^{3}$ \\ Margarita Guenova ${ }^{2,4}$ \\ ${ }^{1}$ Laboratory of Cytogenetics \& Molecular \\ Biology; \\ ${ }^{2}$ Laboratory of Hematopathology \& \\ Immunology; \\ ${ }^{3}$ Clinic of Hematology; \\ ${ }^{4}$ Center of Excellence for Translational \\ Research in Hematology, National \\ Specialized Hospital for Active \\ Treatment of Hematological Diseases, \\ Sofia, Bulgaria
}

\section{Abstract}

We present for the first time a 40 -year-old male patient with a 20 year history of occupational exposure to radiation as a nuclear power plant worker, who developed FIP1L1PDGFRA-positive chronic eosinophilic leukemia 27 months after radiotherapy for testicular seminoma. After an one-year history of dry cough, itching and night sweats, the patient presented with an elevated leukocyte count with absolute eosinophilia of $14.2 \times 10^{9} / \mathrm{L}$, bone marrow and lymph node involvement. Treatment with Imatinib was initiated, resulting in complete hematological remission at the sixth month and complete molecular response by nested primers reverse transcription polymerase chain reaction - at the end of the first year. This case contributes to the clinical heterogeneity of a rare entity such as FIP1L1-PDGFA-positive myeloproliferative neoplasms, and for the possible role of occupational and therapeutic radiation, raising the question if one or both of them might be the causative factor.

\section{Introduction}

The fusion gene FIP1L1-PDGFRA, containing regions of FIP1-like 1 (FIP1L1) gene and the Platelet-Derived Growth Factor Receptor Alpha (PDGFRA) gene as a result of the interstitial cryptic deletion on chromosome $4 \mathrm{q} 12$ [ $\operatorname{del}(4)(\mathrm{q} 12 \mathrm{~g} 12)]$, is considered a recurrent molecular abnormality in patients with eosinophilia-associated myeloproliferative neoplasms (MPNs). The hymeric product of
FIP1L1-PDGFRA acts as a constitutively active tyrosine kinase, however the precise underlying molecular mechanisms of FIP1L1PDGFRA-mediated malignant transformation are still incompletely understood. ${ }^{1}$

According to the revised World Health Organization (WHO) Classification of Tumors of Hematopoietic and Lymphoid Tissues (2008), neoplasms bearing the FIP1L1PDGFRA rearrangements are defined as a rare, separate disease entity. Clinically, the disease is manifested most often as chronic eosinophilic leukemia (CEL), but also as acute myeloid leukemia (AML) or T lymphoblastic lymphoma, or both simultaneously. ${ }^{2}$ As a rule, FIP1L1-PDGFRA-positive MPNs are presented as de novo malignancy, and only two cases following cytotoxic chemotherapy have been reported as therapy-related so far. ${ }^{2,3}$ To our knowledge, FIP1L1-PDGFRA-positive disease after radiation exposure has not been published yet.

In this study we present for the first time a patient with a 20 year history of occupational exposure to radiation, who developed FIP1L1PDGFRA-positive CEL 27 months after radiotherapy for testicular seminoma.

\section{Case Report}

A 40-year-old male nuclear power plant worker was referred to The National Hospital for Active Treatment of Hematological Diseases in Sofia (September 2009) for diagnostic evaluation of leucocytosis and eosinophilia, detected ten months before his admission to our hospital, and resistant to corticosteroid therapy. The patient had a oneyear history of dry cough, predominantly at night, itching and night sweats. Previous medical history included testicular seminoma, diagnosed 27 months earlier (June 2007) and treated with surgery followed by irradiation to the para-aortic and high iliac lymph nodes with a total dose of 36 Gy. No hematological abnormalities were observed during the whole period of regular clinical and laboratory follow up thereafter until December 2008, when the routine blood tests revealed eosinophilia (23\% - $1.3 \times 10^{9} / \mathrm{L}$ ) with a normal white blood cell (WBC) count $\left(5.6 \times 10^{9} / \mathrm{L}\right)$, which progressively increased. On physical examination the patient had no hepato- or splenomegaly, but a single enlarged cervical lymph node. Radiological chest studies showed signs of minor interstitial pulmonary fibrosis in the hilar regions and no lung infiltration.

At the time of admission, laboratory tests revealed elevated WBC count of $18.1 \times 10^{9} / \mathrm{L}$, hemoglobin level of $128 \mathrm{~g} / \mathrm{L}$ and platelet count of $290 \times 10^{9} / \mathrm{L}$. Peripheral blood differential
Correspondence: Gueorgui Balatzenko, Laboratory of Cytogenetics \& Molecular Biology, National Specialized Hospital for Active Treatment of Hematological Diseases, 6, Plovdivsko pole Str., 1756, Sofia, Bulgaria.

Tel. +359.2.97.01.132 - Fax: +359.2.37.01.107

E-mail: balatzenko@hotmail.com

Key words: chronic eosinophilic leukemia FIP1L1-PDGFRA; therapy-related myeloproliferative neoplasm; radiation exposure.

Acknowledgements: this study was supported by a grand from the National Research Fund, Bulgarian Ministry of Education, Youth and Science (CVP-119/2009-D002-35/09).

Received for publication: 14 June 2011.

Accepted for publication: 15 September 2011.

This work is licensed under a Creative Commons Attribution NonCommercial 3.0 License (CC BYNC 3.0).

CCopyright G. Balatzenko et al., 2011

Licensee PAGEPress, Italy

Hematology Reports 2010; 3:e17

doi:10.4081/hr.2011.e17

showed $78 \%$ eosinophils $\left(14.2 \times 10^{9} / \mathrm{L}\right), 14 \%$ mature neutrophils $\left(2.5 \times 10^{9} / \mathrm{L}\right), 2 \%$ basophils $\left(0.36 \times 10^{9} / \mathrm{L}\right)$, and $6 \%$ lymphocytes $\left(1.1 \times 1^{9} / \mathrm{L}\right)$. Laboratory chemistry tests were within normal ranges. On aspirate smears bone marrow was markedly hypercellular due to a proliferation of abnormal eosinophils accounting for $51 \%$ of all bone marrow cells, including $31.5 \%$ eosinophilic myelocytes and $19.5 \%$ mature eosinophils. Most of the cells showed sparse granulation with clear areas of cytoplasm, some had pathological violet granules on Giemsa stains (Figure 1/A). Eosinophils were positive for myeloperoxidase and were negative for non-specific esterase and toluidine blau stains. Neutrophils were $7.5 \%$ of all cells, erythroblasts - $37.5 \%$, small non-granular blasts were $1 \%$ and megakaryocytes were reduced. Immunophenotyping on bone marrow aspirate was performed by flow cytometry using three color combinations of commercially available fluorescent dye labeled monoclonal antibodies (Becton Dickinson) and erythrocyte lyse and wash technique according to manufacturer's instructions. Samples were acquired and analyzed on a BD FACSCanto II flowcytometer and FACSDiva software (Becton Dickinson). Expansion of eosinophils with high FSC and SSC and high expression of CD45 was found, that were positive for CD13, CD33, and CD15, and negative for CD34, CD117, CD64, CD14, and CD10, as well as for major lymphoid lineage associated markers. No aberrant antigen expression was detected. Blast cells were identified, accounting for 


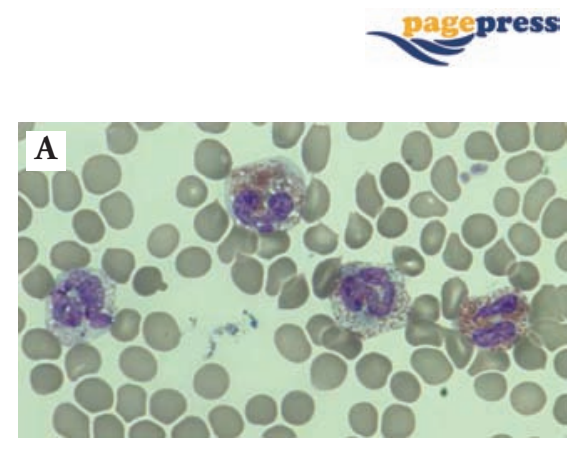

$2.6 \%$ of bone marrow cells showing myeloid phenotype - positive for CD34, CD33, CD13, CD117, CD38. Lymphoid populations were within the normal ranges. An excision biopsy of an enlarged cervical lymph node was performed in order to exclude a concomitant lymphoid neoplasm. The microscopic examination revealed nodal architecture almost completely effaced. Though there was a proliferation of preserved follicles with activated germinal centers, interfollicular areas showed massive infiltration by eosinophils at various stages of maturation as well as CD68-positive histiocytes and densely proliferating small vessels confirmed by immunohistochemistry for CD34 (Figure 1/B). Only few cells showed positive immunostaining for Mast cell tryptase. Unfortunately, at the time of admission the cytogenetic study was unsuccessful. The subsequent analyses during the course of treatment revealed normal karyotype. Molecular analysis on RNA, extracted from bone marrow and peripheral blood cells, using Reverse Transcription Polymerase Chain Reaction (RT-PCR) revealed FIP1L1-PDGFRA rearrangement (Figure 1/C). Screening for $B C R-A B L$ rearrangements and JAK2 gene V617F mutation was negative. Taking into account all above mentioned data, a diagnosis of chronic eosinophilic leukemia with FIP1L1PDGFRA rearrangement was made and Imatinib treatment initiated with a daily dose of $100 \mathrm{mg}$. Normal WBC $\left(4.3 \times 10^{9} / \mathrm{L}\right)$ and eosinophil counts (1\%) were achieved at the second month of therapy, however the bone marrow aspirates were dry until 6 months when a significantly hypocellular bone marrow with a complete remission was found. No FIP1L1-PDGFRA mRNA was detected by a single round RT-PCR and at the end of the first year a complete molecular response by nested primers RT-PCR was registered.

\section{Discussion}

Secondary malignancies are the most serious complications of otherwise successful treatment of some solid tumors. Myelodysplastic syndromes (MDS) and AML are the most common therapy-related hematological malignancies and a large amount of data is available regarding their epidemiology, molecular pathogenesis, clinical behavior and response to therapy. Secondary MPNs are significantly rarer. Almost all of these cases, reportedly more than 150 patients, present as secondary $B C R-A B L$-positive chronic myelogenous leukemia (CML). ${ }^{4}$ In contrast, secondary $B C R-A B L$-negative MPNs have only been occa- sionally observed. In a survey among 29,356 testicular cancer survivors, 621 secondary cancers were found, and the only MPNs were four cases of CML. ${ }^{5}$ Similarly, in another study, a review of 463,618 cases of cancer patients treated with chemotherapy and radiotherapy, revealed 233 patients with hematological malignancies including AML/MDS ( $n=741)$, CML $(n=178)$, chronic lymphocytic leukemia (CLL) $(n=253)$ and acute lymphoblastic leukemia ( $\mathrm{n}=61)$. Notably, no cases of $B C R$ - $A B L$-negative MPNs were identified. ${ }^{5}$

Herein, we report a patient who developed a secondary FIP1L1-PDGFRA-positive CEL, 27 months after radiotherapy for testicular seminoma and 20 years of occupational exposure at a power plant station. This case is of clinical interest in several aspects. Firstly, although more than a hundred cases of FIP1L1-PDGFRA-positive MPNs have been reported so far, only two of them were secondary to chemotherapy for a primary cancer. ${ }^{3,6}$ In the first case, FIP1L1-PDGFRA fusion gene was detected 11 years after 3 courses of combination chemotherapy because of non Hodgkin's lymphoma and 3 years after therapy with cyclophosphamide for hypereosinophylic syndrome. ${ }^{3}$ In the second case, the molecular abnormality was found 1 year after the application of several courses of multiagent chemotherapy for Langerhans cell histyocytosis. ${ }^{6}$ No radiotherapy-related FIP1L1-PDGFRApositive MPN has been reported so far. Secondly, our patient has two radiation exposure events - occupational and therapeutic, raising the question if one or both of them might be the causative factor. Though it is well known that abdominal and pelvic irradiation may produce eosinophilia, two facts are against the hypothesis that radiotherapy has a primary role in the secondary malignancy pathogenesis. In the reported case, the diagnosis of CEL was made only 27 months after radiotherapy and this period was significantly shorter than the 5-9 years or longer, reported in the literature, though secondary myeloid malignancies have been reported after similar or even shorter latency period. ${ }^{7}$ Besides, our patient received a high dose of $36 \mathrm{~Gy}$, though for a larger volume including parts of the abdomen and pelvis and the radiation-related leukemia risk has proven to be considerably lower at high doses due to cell killing. ${ }^{8}$

On the other hand, the patient's long-term occupational radiation exposure may not be the only culprit as many studies have failed to prove greater risk for hematological neoplasms among nuclear power plant workers compared with national rates. ${ }^{9}$ Similarly, no strong evidence for increased risk of leukemia, or other malignant disease was
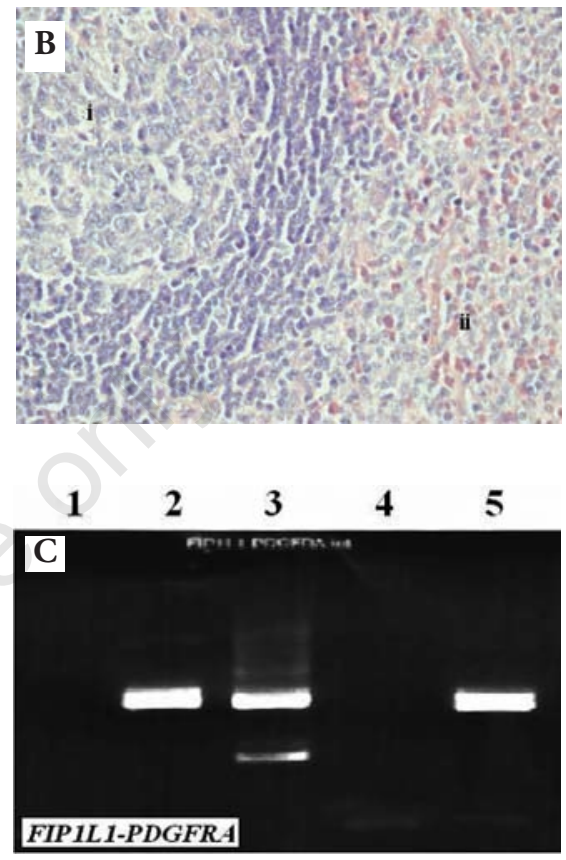

Figure 1. Morphological and molecular characteristics of the reported patient with FIP1L1-PDGFRA-positive chronic eosinophilic leuekemia: a) blood smear. Increased number of abnormal eosinophils in the field, characterized with sparse granulation and clear areas of cytoplasm, some with pathological granules. (WrightGiemsa stain, x 1000); b) a biopsy of an enlarged cervical lymph node. Nodal architecture almost completely effaced. Though there was a proliferation of preserved follicles with actived germinal centres i), interfollicular areas ii) showed massive infiltration by eosinophils, histiocytes and densely proliferating small vessels. (Hematoxylin-eosin staining, $x$ 400); c) positive result for FIPIL1-PDGFRA fusion transcripts after nested primers PCR on cDNA using primers pairs i) FIP1L1(ext)-F: acctggtgctgatctttctgat / PDGFRA (ext)-R: tgagagcttgttttcactgga and ii) FIP1L1 (int)-F: aagaggatacgaatgggacttg /PDGFRA (int) R: gggaccggcttaatccatag: ${ }^{1}$ No template control; ${ }^{2}$ Direct amplification of patient's cDNA using internal primers; ${ }^{3}$ Re-amplification of patient's first round PCR product using internal primers; ${ }^{4}$ Negative control cDNA of a healthy person; ${ }^{5}$ Positive control - EOL-1 cell line. 
found among adult populations with low doserate fractioned exposures after the Chernobyl accident. ${ }^{10}$ However, the understanding of radiation biology has undergone a fundamental shift in paradigms away from deterministic hit-effect relationships and towards complex ongoing cellular responses such as increased genomic instability, and decreased adaptive responses observed at very low doses, and particularly relevant when exposure is spread over a period of time. These are circumstances that are important to understanding cancer risk associated with occupational radiation exposures due to increased sensitivity to physical, radiation and chemical agents and modified biological effects. ${ }^{11}$ Therefore, in accordance with the hypothesis of two/multiple-hits carcinogenesis, ${ }^{12}$ it seems more probable that in our patient, the cumulative dose or sequential exposure to both occupational and therapeutic radiation have contributed to the development of a FIP1L1-PDGFRA-positive neoplasm. However, a question still remains why no other similar cases have been reported so far in thousands of occupational radiation workers as well as in patients who have undergone radiation therapy.

Several factors might be related to the absence of reported cases of FIP1L1PDGFRA(+) CEL. Firstly, CEL in general is a very rare disease. ${ }^{2}$ Besides, FIP1L1-PDGFRA fusion gene has been discovered not long ago ${ }^{13}$ and cannot be detected by conventional cytogenetics, therefore, the respective abnormality had not been tested routinely in a significant proportion of patients and the true incidence of FIP1L1-PDGFRA-positive cases is still unknown. To our knowledge there is at least one case of CEL, developed 6 years after radiotherapy for thyroid cancer, reported so far. Interestingly, this patient was positive for $\mathrm{t}(6 ; 11)(\mathrm{q} 27 ; \mathrm{q} 23) / M L L-A F 6$, which is a typical molecular marker of AML, but at that time FIP1L1-PDGFRA-rearrangement was not tested. ${ }^{14}$ On the other hand, the risk of develop- ment of secondary malignancies after radiation exposure depends on a number of factors - cumulative radiation dose received, the individual genetic background, exposure to additional mutagenic factors, etc. that might vary from case to case.

In conclusion, this case contributes to the clinical heterogeneity of a rare entity such as FIP1L1-PDGFA - positive myeloproliferative neoplasms, and for the possible role of occupational and therapeutic radiation, raising the question if one or both of them might be the causative factor.

\section{References}

1. Buitenhuis M, Verhagen LP, Cools J, Coffer PJ. Molecular mechanisms underlying FIP1L1-PDGFRA-mediated myeloproliferation. Cancer research 2007;67:375966.

2. Bain B, Gilliland DG, Horny HP, Vardiman JW. Myeloid and lymphoid neoplasms with eosinophilia and abnormities of PDGFRA, PDGFRB and FGFR1. In: Swerdlow SH, Campo E, Harris HL, Jaffe ES, Pileri SA, Stein H, Thele J, Vardiman JW, editors. WHO classification of tumors of haematopoieitic and lymphoid tissues. Lyon: IARC, 2008; pp.68-73.

3. Tanaka Y, Kurata M, Togami K, et al. Chronic eosinophilic leukemia with the FIP1L1-PDGFRalpha fusion gene in a patient with a history of combination chemo-therapy. Int J Hematol 2006;83: 152-5.

4. Lichtman MA. Is there an entity of chemically induced BCR-ABL-positive chronic myelogenous leukemia? Oncologist 2008;13: 645-54.

5. Schairer C, Hisada M, Chen BE, et al. Comparative mortality for 621 second cancers in 29356 testicular cancer survivors and 12420 matched first cancers. J Natl
Cancer Inst 2007;99:1248-56.

6. Ohnishi H, Kandabashi K, Maeda Y, et al. Chronic eosinophilic leukemia with FIP1L1-PDGFRA fusion and T6741 mutation that evolved from Langerhans cell histiocytosis with eosinophilia after chemotherapy. Br J Haematol 2006; 134: 547-9.

7. Mangano JJ. A short latency between radiation exposure from nuclear plants and cancer in young children. Int $\mathrm{J}$ Health Serv 2006;36:113-35.

8. Travis LB. The epidemiology of second primary cancers. Cancer Epidemiol Biomarkers Prev 2006;15:2020-6

9. Cardis E, Vrijheid M, Blettner M, et al. The 15-Country Collaborative Study of Cancer Risk among Radiation Workers in the Nuclear Industry: estimates of radiationrelated cancer risks. Radiation Research 2007;167:396-416.

10. Moysich KB, Menezes RJ, Michalek AM. Chernobyl-related ionizing radiation exposure and cancer risk: an epidemiological review. The Lancet Oncology. 2002; 3:269-79.

11. Pelevina II, Afanasjev GG, Gotlieb VY, Konradov AA. Twenty years after the Chernobyl accident - Is it much or not? In: Burlakova E, Naidich V, editors. 20 Years after the Chernobyl accident. Hauppauge, NY: Nova Science Publishers, Inc; 2006: pp. 331-343.

12. Kralovics R. Genetic complexity of myeloproliferative neoplasms. Leukemia 2008; 22:1841-8.

13. Cools J, DeAngelo DJ, Gotlib J, et al. A tyrosine kinase created by fusion of the PDGFRA and FIP1L1 genes as a therapeutic target of imatinib in idiopathic hypereosinophilic syndrome. N Engl J Med 2003; 348:1201-14.

14. Suzuki S, Chiba K, Toyoshima N, et al. Chronic eosinophilic leukemia with $\mathrm{t}(6 ; 11)(\mathrm{q} 27 ; \mathrm{q} 23)$ translocation. Ann Hematol 2001;80:553-6. 